\title{
Der 24. Kongress der EADV in Dänemark
}

\author{
Vom 7. bis 11. Oktober 2015 fand der Jahreskongress der \\ European Academy of Dermatology and Venereology (EADV) \\ in Kopenhagen/Dänemark statt. Mit rund 700 Referenten aus \\ 30 Ländern und knapp 10000 Besuchern ist er die größte \\ Tagung für Dermatologen in Europa.
}

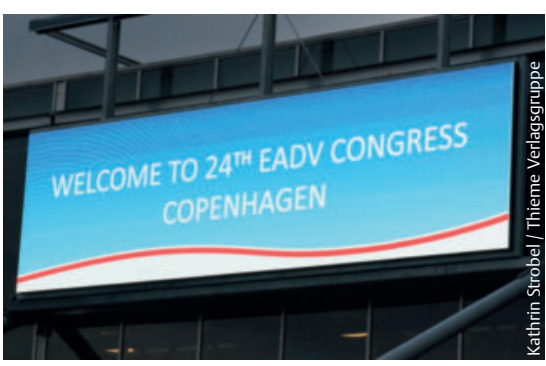

Das wissenschaftliche Programm gliederte sich in 41 Themenkomplexe, die in insgesamt 180 Sessions behandelt wurden. Exemplarisch sollen hier einige der Themen beleuchtet werden.

\section{Melanom - die Risikofaktoren (er-)kennen \\ $\nabla$}

Etwa 65\% der Melanome sind auf UV-Exposition zurückzuführen. Doch was ist mit den restlichen 35\%? „Die Entstehung von Melanomen ist multifaktoriell bedingt. Zahlreiche biologische, kulturelle und geographische Faktoren wirken zusammen, die außerhalb unserer Kontrolle liegen“, so Prof. Veronique del Marmol, Brüssel/Belgien, im Rahmen der Fachpressekonferenz. Etwa 10\% sind das Resultat von Keimbahnmutationen. Mutationen in bestimmten Signalwegen (wie z.B. BRAF, NRAS, MITF) können Krebs auslösen. Die dahintersteckenden Mechanismen sind jedoch noch unklar.

Eine weitere typische Eigenschaft von Melanomen ist eine Überexpression von nichtkodierender RNA (wichtigster Vertreter: miRNA). MiR-21 ist eine der ersten miRNAs, die mit Krebs assoziiert werden. Eine miR-21-Überexpression kann durch UV-Bestrahlung, aber auch durch giftige Chemikalien, Luftverschmutzung, Rauchen, chronische Entzündungen, einen westlichen Ernährungsstil sowie Adiposi- tas hervorgerufen werden. Diese können daher als potenzielle Risikofaktoren für die Melanombildung betrachtet werden. Auch Kuhmilch könnte einen Risikofaktor darstellen: Ein Großteil des darin enthaltenen miR-Anteils ist miR-21. „Obwohl nicht-UV-induzierte Melanome selten sind, müssen wir uns darüber bewusst sein, dass es neben der UV-Exposition noch weitere Risikofaktoren, wie z.B. die Lebensführung, für die Melanomentstehung gibt“, so del Marmol.

Auch in der gemeinsamen Session von EUROMELANOMA und der European Association of Dermato-Oncology wurde die Identifikation von Risikofaktoren thematisiert. In ihrem Vortrag stellte Prof. Gaëlle Quéreux, Nantes/Frankreich, ein Werkzeug zur gezielten Bestimmung des individuellen Melanom-Risikos vor. Mithilfe eines Fragebogens und des Self-Assessment of Melanoma Risk Scores (SAMScore) sollen Patienten ohne zusätzliche ärztliche Hilfe ihr eigenes Risikolevel ermitteln. Auf diese Weise könnten (Hoch-) Risikopatienten identifiziert und Risikomodelle erarbeitet werden.

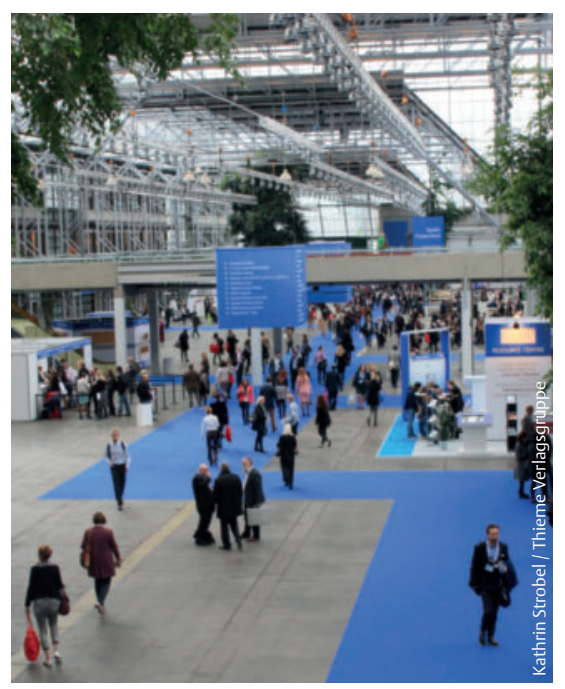

Medikamentenresistente Gonorrhö - noch zu stoppen? $\nabla$

Sexuell übertragbare Infektionen sind ein großes Problem. Weltweit infizieren sich jährlich fast 400 Mio. Menschen zwischen 15 und 49 Jahren mit Chlamydia trachomatis (146 Mio.), Neisseria gonorrhoea (51 Mio.), Syphilis (5 Mio.) oder Trichomonas vaginalis (239 Mio.). Aktuell ist es vor allem die Gonorrhö (syn.: Tripper), die Prof. Colm O'Mahony, Chester/England, Sorgen bereitet: „Neisseria gonorrhoea hat inzwischen gegen alle Antibiotika Resistenzen entwickelt, die bislang dagegen eingesetzt wurden“, erklärte er im Rahmen der Fachpressekonferenz.

Allein im Großraum Leeds im Norden Englands wurden zu Kongressbeginn 11 neue Fälle gemeldet. Eine weltweite Verbreitung des resistenten Erregerstamms sei laut O'Mahony unvermeidbar; trotzdem werde alles versucht, um diese zumindest zu verlangsamen. Der wichtigste (und momentan einzig wirksame) Ansatz zur Prävention einer weltweiten Epidemie seien die Aufklärung und der Aufruf zu geschütztem Sex. Zudem würden neue Wirkstoffe zur Bekämpfung von N. gonorrhoea getestet. Bis eine neue Therapieoption entwickelt sei, gebe es jedoch keine Lösung.

Auch Syphilis-Infektionen können inzwischen nicht mehr mit der bisher verwendeten Standardtherapie Azithromycin behandelt werden. Die einzige noch verbleibende Alternative ist die aufwendigere und kostspieligere Injektionstherapie mit Penicillin. Diese ist zwar wirksam, doch nicht bei allen Patienten anwendbar: Wer unter einer Penicillin-Allergie leidet, kann nicht behandelt werden.

\section{Tätowierungen und Piercings - Body Art ist nicht ohne Risiken $\nabla$}

Tätowierungen und Piercings sind in der Mainstreamkultur angekommen und beschränken sich längst nicht mehr nur auf bestimmte Bevölkerungs- oder Altersgruppen. So sind etwa $10 \%$ der Gesamtbevölkerung und ca. 30\% der über 40-jährigen tätowiert und/oder gepierct. Ohne Frage: Tätowierungen und Piercings sind in. Doch Body Art ist nicht ohne Risiken. Die Hauptkomplikationen: Infektionen und Allergien. Bei Piercings seien v.a. Nickelallergien, trotz des in der EU geltenden Nickelverbots für Schmuck, noch weit 
verbreitet, sagte Kongresspräsidentin Dr. Christa de Cuyper, Brügge/Belgien, im Rahmen der Fachpressekonferenz. Neben den Hygienestandards der Tätowierungsstudios seien als kritische Faktoren bei Tätowierungen v.a. die verwendeten Tattoofarben zu nennen. Zwar gibt es Regulationen bezüglich der Inhaltsstoffe, doch bislang ist nur wenig über die Wirkung von Farbpigmenten auf den Körper bekannt. Eine Positivliste bedenkenlos zu verwendender Tattoofarben gibt es daher noch nicht. Besonders problematisch seien rote Farben sowie Henna, so de Cuyper. Weitere in Tattoofarben enthaltene gefährliche Inhaltsstoffe sind z. B. karzinogene aromatische Amine, Schwermetalle, Konservierungsmittel und weitere Stoffe, deren Funktion völlig unklar ist, wie z.B. Hexachlorbenzol, Schellac und essenzielle Öle. Man dürfe nicht vergessen, dass die Basis der meisten Tattoofarben Industriefarbstoffe sind. Dies müsse sich dringend ändern. „Wir brauchen einen einheitlichen europäischen Standard“, so de Cuyper.

Auch die Tattoo-Entfernung birgt Risiken: Selbst moderne Lasertechnologien garantieren kein perfektes Ergebnis. Häufig kommt es zu Depigmentierungen und/ oder Narbenbildung. Beim Abbau von Tattoofarbstoffen durch eine Laserbehandlung können zudem potenziell schädliche Stoffe wie z.B. Blausäure entstehen. Um das öffentliche Bewusstsein für die mit Tätowierungen und Piercings verbundenen Risiken zu stärken, hat die EADV eine Kampagne gestartet: Unter dem Titel „Body Art is not without Risk! “ werden Informationen und weiterführende Literatur zum Thema angeboten.

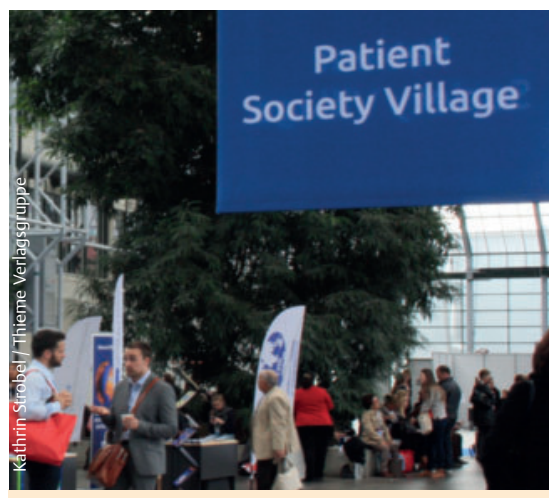

Eine Besonderheit war die aktive Einbindung von Patientenverbänden. Im „Patient Village“ waren u.a. die European Umbrella Organisation for Psoriasis Movements (EUROPSO), die Global Allergy \& Asthma Patient Platform (GAAPP) und die International Federation of Psoriasis Associations (IFPA) vertreten.

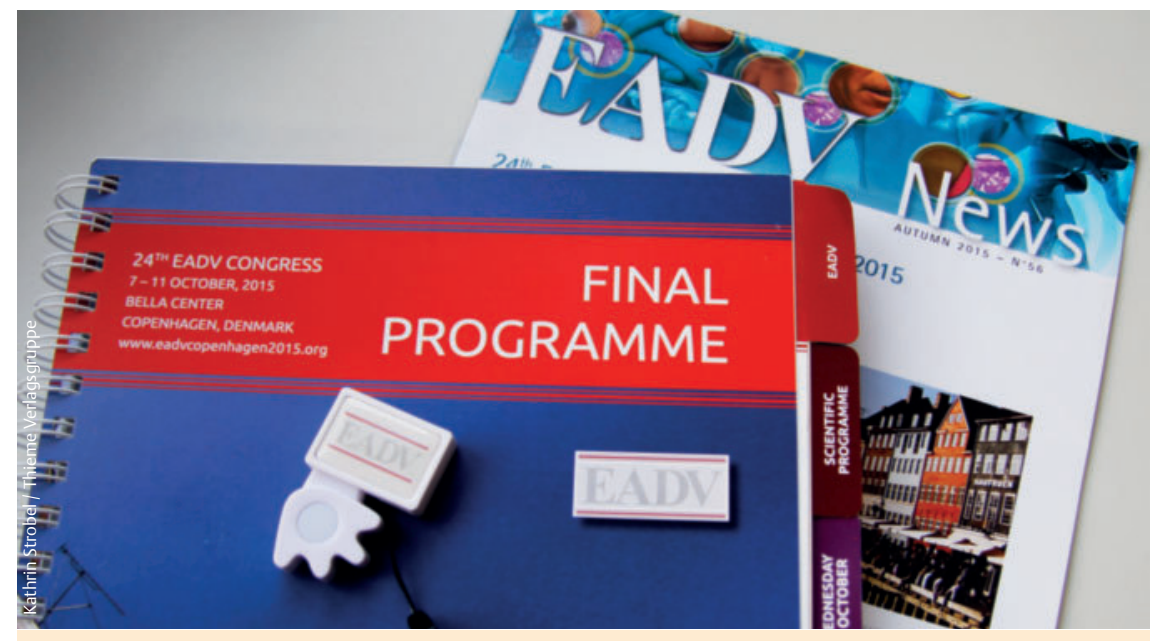

Datenaustausch leicht gemacht: Jeder Kongressbesucher erhielt bei der Anmeldung einen Poken. Auf dem USB-Speicher in Handform waren die bei der Akkreditierung im Voraus angegebenen Kontaktdaten gespeichert. Durch Aneinanderlegen zweier Pokens wurden die Kontaktdaten der Besitzer über Nahbereichsfunk ausgetauscht oder Abstracts einer Veranstaltung gespeichert. Bei erfolgreicher Übertragung leuchten die Hände grün.

\section{Die Bedeutung der Dermatologie für die moderne Medizin $\nabla$}

„Die Forschungen und Erkenntnisse im Bereich der Dermatologie reichen weit über das Fachgebiet heraus“, so Prof. Martin Röcken, Tübingen. So eröffnen die Fortschritte in der Entwicklung neuer Wirkstoffe zur Behandlung der Psoriasis (z.B. Interleukin[IL]-17- und IL-23-Inhibitoren) auch für andere Erkrankungen neue Therapieoptionen - z. B. für die entzündliche Darmerkrankung, rheumatoide Arthritis und multiple Sklerose. Ähnliches gilt für die Entwicklungen im Bereich der Hautkrebsforschung: Die Anti-PD1-Therapie ist die erste effiziente Immuntherapie zur Krebsbekämpfung. Die positiven Erfahrungen, die damit in der Behandlung des metastasierenden Melanoms gemacht wurden, ließen sich vermutlich auch auf andere Krebsarten ausweiten, so Röcken. Die neuen Therapieoptionen seien jedoch sehr kostspielig. Sie flächendeckend für einen großen Teil der Patienten verfügbar zu machen, sei eine große gesundheitspolitische Herausforderung.

Die Bedeutung der Dermatologie für die restliche Medizin werde auch eindrücklich durch die diesjährigen Nobelpreise widergespiegelt. So erhielten William C. Campbell und Satoshi Ōmura den Nobelpreis für Medizin für ihre Forschung zur Bedeutung der Haut für die Infektiologie. Tomas Lindahl, Paul Modrich and Aziz Sancar wurden für die Erforschung der Mechanismen ausgezeichnet, die von Zellen zur DNA-Reparatur eingesetzt werden. Ihre Ergebnisse stellen einen ent- scheidenden Schritt für das Verständnis der Pathogenese von Hautkrebs und Hautalterung dar.

\section{Innovative Programmgestaltung \\ $\checkmark$}

Eine der Besonderheiten des Kongresses war die Staffelung des wissenschaftlichen Programms nach verschiedenen Schwierigkeitsgraden bzw. Wissensniveaus:

- Training and Educational Forum

- Review and Updates

- Expert Forum

Wie der Vorsitzende des Scientific Programming Committees, Prof. Röcken, erklärte, erhielt jedes der Hauptthemen des Programms, wie z.B. Hautkrebs, Allergien, Entzündungen, infektiöse Erkrankungen, ein halb- bis ganztägiges Zeitfenster. Der Ablauf der darin enthaltenen Sessions folgte einer einheitlich festgelegten Struktur: von Klinik über Dermato-Pathohistologie und Epidemiologie zu Diagnosen und Differenzialdiagnosen, gefolgt von der Vorstellung der verfügbaren Therapieoptionen. Die Schwierigkeitsniveaus waren farblich gekennzeichnet.

\section{Auf ein Wiedersehen in Wien \\ $\nabla$}

Der nächste Jahreskongress der EADV wird vom 28. September bis 2. Oktober 2016 im Austria Center in Wien stattfinden.

Kathrin Strobel, Mannheim 